\title{
The Effect of Ankle Angle and Foot-plate Length of Ankle-Foot Orthoses on Spatiotem- poral Parameters and Knee Joint Angle in Post-Stroke Hemiplegic Gait
}

\author{
Razieh Didevara ${ }^{1}$, Gholamreza Aminian*2, Aliyeh Daryabor ${ }^{1,3}$
}

1. MSc. Of Prosthetics and Orthotics, Department of Orthotics and Prosthetics, University of Social Welfare and Rehabilitation Sciences, Tehran, Iran

2. Associate Professor, Department of Orthotics and Prosthetics, University of Social Welfare and Rehabilitation Sciences, Tehran, Iran

3. PhD Student Of Prosthetics and Orthotics, Department of Orthotics and Prosthetics University of Social Welfare and Rehabilitation Sciences, Tehran, Iran

\begin{tabular}{c} 
Article Info \\
\hline Received:2019/01/15; \\
Accepted:2019/03/08; \\
Published Online: $2019 / 03 / 20$ \\
DOI: 10.30699/fdisj.2.1.37 \\
How to Cite This Article \\
Didevara R., Aminian Gh., \\
Daryabor A. The Effect of \\
Ankle Angle and Foot-plate \\
Length of Ankle-Foot Or- \\
thoses on Spatiotemporal. \\
Function and Disability Jour- \\
nal. 2019 (Winter). Vol:2. \\
No:1. pages:37-45
\end{tabular}

Use your device to scan and read the article online

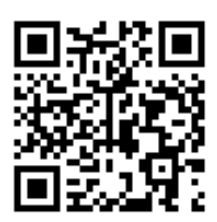

\section{ABSTRACT}

Background and Objectives: Equinovarus deformity and knee hyperextension are the most common problems in stroke patients. Ankle foot orthosis (AFO) is commonly prescribed to improve these disorders in these patients, as well as gait parameters that are approved. The aim of this study is to investigate the effect of ankle alignment and rigid AFO footplate length on spatiotemporal parameters and knee joint angle in hemiplegic post-stroke patients.

Materials and Methods: This quasi-experimental study was performed on 6 hemiplegic post-stroke patients using three rigid AFO modes, including neutral ankle angle with full-length foot-plate -conventionally aligned AFO (CAFO), neutral ankle angle with $3 / 4$ length foot plate $(3 / 4 \mathrm{AFO})$, and $5^{\circ}$ dorsi-flexion ankle angle with full-length foot-plate $\left(5^{\circ} \mathrm{DF} \mathrm{AFO}\right)$ to investigate the effect of the alignment and foot plate length on spatiotemporal parameters and knee joint angle. The 3D motion analysis system with a Vicon Camera was used to collect the spatiotemporal parameters data.

Results: The mean walking speed of patients after using $5^{\circ} \mathrm{DF}$ AFO was significantly higher compared to CAFO $(P=0.036)$. The mean stride length, cadence and maximum knee extension angle in the stance phase during single limb support was not significantly different in the three different test modes of $3 / 4 \mathrm{AFO}, \mathrm{CAFO}, 5^{\circ} \mathrm{DF}$ AFO $(P>0.05)$.

Conclusion: The DF AFO with $5^{\circ}$ ankle angle increases the walking speed and also controls knee hypertension in the terminal stance phase, which can be due to the progression of tibia in the mid to late stance phase and the alteration of ground reaction force vector.

Keywords: Stroke, Hemiplegia, AFO, Alignment, Walking speeds, Knee kinematics, Footplate length.

Corresponding information:

Gholamreza Aminian, Associate Professor, Department of Orthotics and Prosthetics, University of Social

Welfare and Rehabilitation Sciences, Tehran, Iran E mail: gholamrezaaminian@yahoo.com

Copyright (C) 2019, Function and Disability Journal. This is an original open-access article distributed under the terms of the Creative Commons Attribution-noncommercial 4.0 International License which permits copy and redistribution of the material just in noncommercial usages with proper citation.

\section{Introduction}

Stroke (cerebrovascular accident, CVA) is a type of acute nerve injury due to abnormalities in the blood supply to part of the brain tissue (Easton et al., 2009). Limb movement asymmetry, difficulty and slowness of movement and reduced walking speed are some re- lated complications (Bovonsunthonchai, Hiengkaew, Vachalathiti, \& Vongsirinavarat, 2011). Moreover, the changes in the gait pattern of patients due to remaining muscle weakness, spasm, and abnormal movements may be seen and these factors may also cause poor balance, increased energy consumption and the 
risk of falling (da Cunha Jr et al., 2002). Improvement of gait pattern and recovery in the ability to walk are the main objectives of rehabilitation in these patients (Danielsson \& Sunnerhagen, 2004). The most widely prescribed orthosis for hemiplegic patients is ankle foot orthosis (AFO). The AFO can prevent the ankle planar flexion in the stance phase, and control the knee hyperextension, indirectly (Hsu, Michael, \& Fisk, 2008). Clinically, keeping the ankle in dorsiflexion about 5 to 10 degrees causes the knee to fit in the proper degree of flexion, reducing the tendency towards genurecurvatum in the stance phase (Chen, Yeung, Wu, \& Chiang, 1998; Eddison, Chockalingam, \& Osborne, 2015; Hung, Chen, Yu, \& Hsieh, 2011). Some researchers like Lehman, Miyazaki, and Oshawa et al. have reported the establishment of the 5, 7 and 10 degrees of dorsiflexion angle in an AFO may control knee hyperextension (Lehmann, Condon, \& Price, 1987; Miyazaki, Yamamoto, \& Kubota, 1997; Ohsawa et al., 1992).

Regardless of the ankle joint alignment that can be effective on the gait parameters, the footplate length of the orthosis might be another variable which can affect the gait of hemiplegic. The most commonly used footplate length in AFO is in full-length or $3 / 4$ length foot-plate (ending proximal to the metatarsal heads). The footplate length seems to affect the second and third rockers of foot and ankle mechanism of these patients during stance phase. Although the number of studies in this area is very limited, only the results of Fatone et al (2009) study showed that full-length footplate articulated AFO in conjunction with a plantar flexion stop might improve early stance knee moments for people with post stroke hemiplegia.

According to the above explanation, therefore, the aim of this study is to investigate the effect of AFO alignment and footplate length on spatiotemporal parameters and knee joint angle in hemiplegic poststroke patients.

\section{Materials and Methods}

This is a quasi-experimental study within a group but without a control group for comparing the effects of the three conditions by using three types AFO. It was approved by the Ethics Committee of the University of Social Welfare and Rehabilitation Sciences with the ethics code, IR.USWR.REC.1395.56

\section{Participants}

According to the sample size formula and by using speed variable in the Andrew study (2002) where the $\mathrm{z}=1.96, \mu 1=0.32 \& \mu 2=0.35$, seven hemiplegic patients participated in this study, however the stuy has had one missing data after data analyzing.

Inclusion criteria for participants were at least six months after stroke, no history of using AFO since the onset of the stroke, no major involvement or difficulty in the contra lateral side, ability to walk independently without cane and walker, maximum grade 2 in ankle plantar flexion muscles spasticity in terms of Ashworth scale and the presence of knee hyperextension in the stance phase when the knee extension is zero degrees in the ipsilateral during the stance phase (Lamontagne, Malouin, \& Richards, 2001). Exclusion criteria included people with hammer toes deformity, hip and knee contracture, knee hyperflexion, and patients with cardiorespiratory disorders. The participants were selected from hemiplegic patients referring to Imam Khomeini Hospital and the tests were performed in Javad Movafaghian Research Center in Tehran (Iran). First of all, the procedure of tests was fully explained to the patients, and the subjects who met the study inclusion and exclusion criteria with full consent were enrolled in the study.

\section{Intervention}

To construct the orthosis, the below-knee casting and molding was done according to Sumiya, Suzuki, \& Kasahara (1996) for each patient in a position where the person was sitting on the chair and the foot in the semi-weight bearing position, so that the ankle was on the angle of 90 degrees and the shin on both frontal and sagittal planes in perpendicular position and 5 degree of dorsi-flexion for other condition by using goniometry. In the next step, the plaster mold was modified, including reducing the pressure on the malleoli, correcting the angle of the mold on the ankle, and maintaining the standard alignment of the shin. Finally, three types of rigid AFO with 4-mm 
polypropylene sheet were constructed so that the trim line of all three types was one inch lower than neck of fibula in proximal border and also passed in front of malleloi in the following conditions.

1) Neutral ankle angle and full-length foot-plate, conventionally aligned AFO (CAFO)(Figure 1)

2) Neutral ankle angle and $3 / 4$ length foot-plate $(3 / 4 \mathrm{AFO})$ (Figure 2)

3) $5^{\circ}$ dorsiflexion ankle angle and full-length footplate $\left(5^{\circ} \mathrm{DF}\right.$ AFO) (Figure 3)

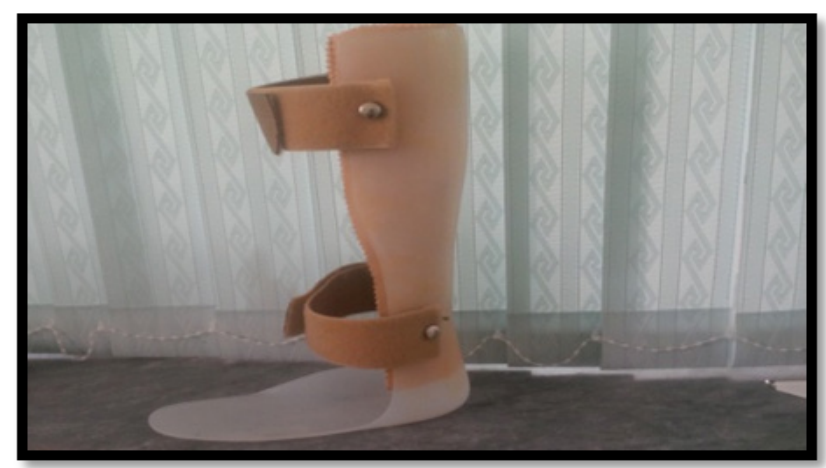

Figure 1. Neutral ankle angle and full-length foot-plate - conventionally aligned AFO(CAFO)

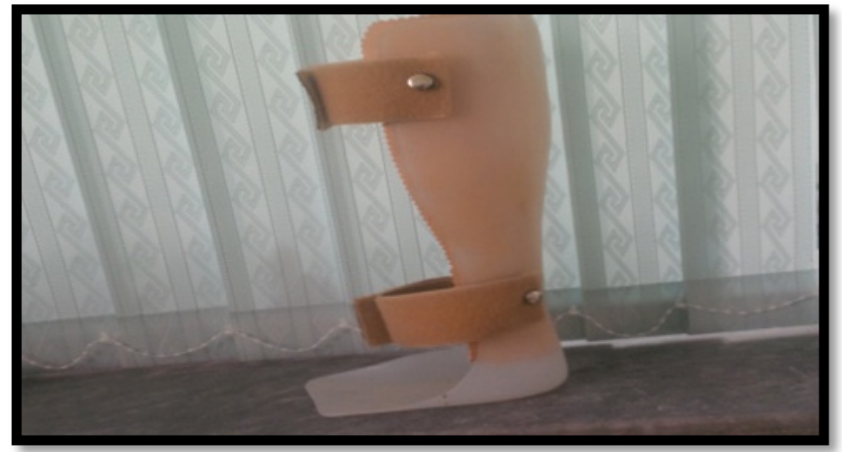

Figure 2. Neutral ankle angle and $3 / 4$ length foot-plate $(3 / 4$ AFO)

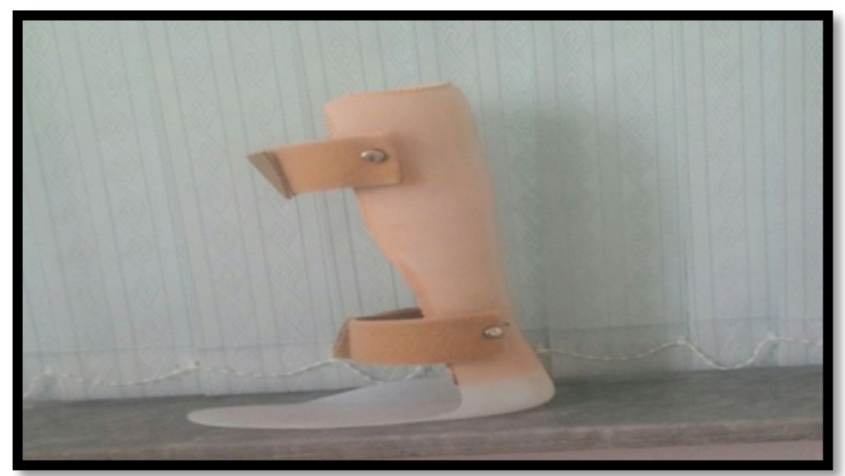

Figure 3. $5^{0}$ dorsiflexion ankle angle and full-length foot-plate $\left(5^{\circ} D F A F O\right)$

\section{Data collection}

After preparing the orthoses, the patients referred to the Ergonomic product testing laboratory at the University of Social Welfare and Rehabilitation Sciences of Tehran. Prior to data collection, subjects were allowed to wear these orthoses 2 weeks of accommodation to every orthotic condition. The $3 \mathrm{D}$ motion analysis system with the Vicon Camera (UK Oxford
Metrics) with six infrared cameras and a frequency of $100 \mathrm{~Hz}$ was used to obtain the necessary information. Markers were attached bilaterally on the calcaneus, lateral and medial malleolus, greater trochanter femur, lateral and medial femur epicondyle, sacrum at the superior aspect of the L5/sacral interface, anterior superior iliac spine (Fatone, Gard, \& Malas, 2009).

All tests were performed for all participants with the 
same shoes (All-Star shoe) with a same heel height. Before the start of the main test, the subject walked in the laboratory for a few minutes for accomodating with different AFO's to meet the conditions (depending on the patient's comfort). During the test, the subject walked in a straight line with a length of 5 meters with their own stride. A walking test was performed for each individual in three different orthotics conditions. Three tests were taken in each condition. The ordering of different situations was done randomly to avoid the effect of the order of the tests on the results. Several minutes of rest were considered for the patient as sitting on the chair.

\section{Stitistical analysis}

Shapiro Wilk test was performed to determine the normal distribution of the data with three different AFO modes. Regarding the normal distribution of variables in walking speed, gait cadence and knee joint angle, the parameters were compared in three different modes of AFO with repeated measures ANOVA test. In the variable stride length, Friedman test was used because of the absences of normal distribution of the data. For data analysis, SPSS version 14 was applied, and the significant level was less than 0.05 .

\section{Results}

The study was conducted on six people with poststroke hemiplegia, including five females and one male, of which two had right hemiplegia and four had left hemiplegia. The demographic characteristics of patients are presented in Table 1 as the mean \pm standard deviation.

\section{Spatiotemporal parameters}

The results of this study showed an increase in the stride length and walking speed of participants with $3 / 4 \mathrm{AFO}$ and $5^{\circ} \mathrm{DF}$ AFO conditions compared to CAFO (Figure 4), and this variation was statistically significant for the walking speed in $5^{\circ} \mathrm{DF}$ AFD $(P=$ 0.036 ), but there was not statistically significant for the stride length $(P>0.05)$. Moreover, the increase in gait cadence was observed for $3 / 4 \mathrm{AFO}$ compared to the CAFO, although it was not statistically significant $(P>0.05)$, see Table 2.

\section{Knee joint kinematics}

In the present study, the maximum knee extension angle in the stance phase during single-limb support for the $3 / 4 \mathrm{AFO}$ and $5^{\circ} \mathrm{DF}$ AFO was respectively decreased in comparison with the CAFO, although these changes were not statistically significant $(P>0.05)$, see Table 2.

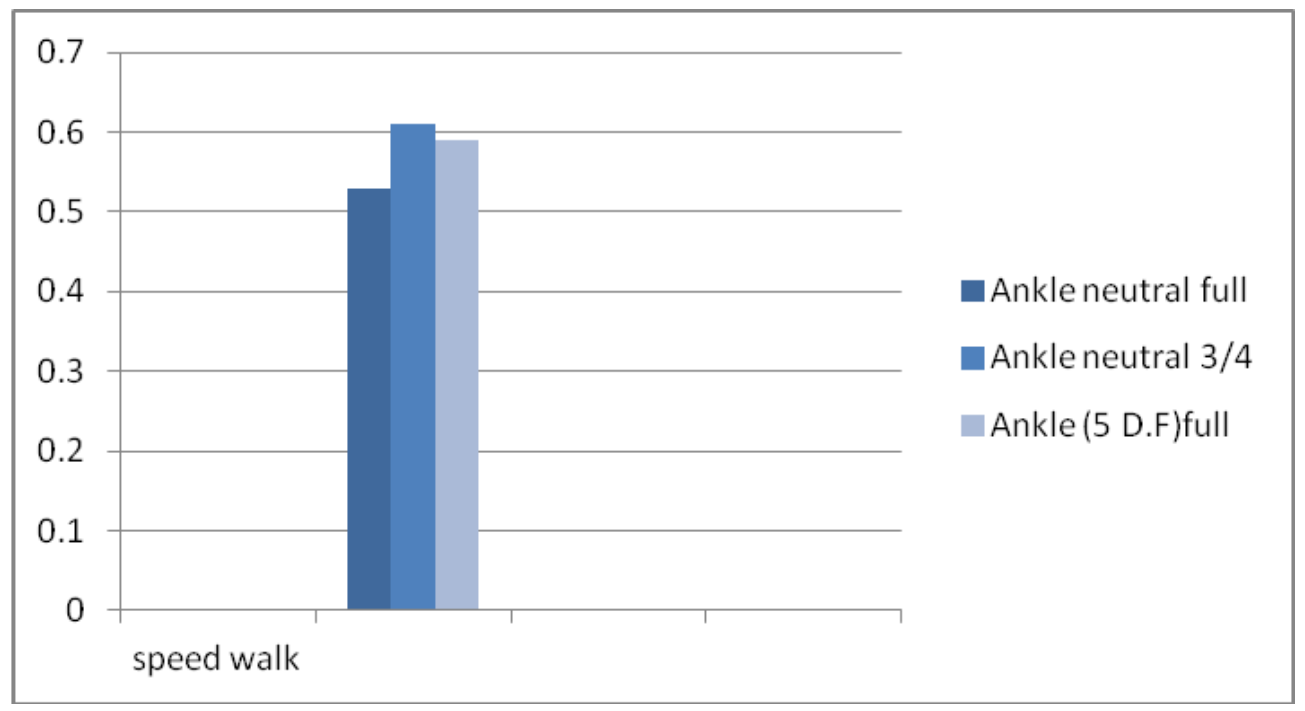

Figure 4. The comparison between mean of speed walk with three different types of AFO 
Table1. Subject's demographic information

\begin{tabular}{cccccccc} 
Subject & Gender & Affected side & Weight(kg) & height $(\mathrm{cm})$ & Age(year) & $\begin{array}{c}\text { Time since } \\
\text { onset (year) }\end{array}$ & $\begin{array}{c}\text { Modified } \\
\text { Ashworth Scale }\end{array}$ \\
\hline 1 & Female & Right & 55 & 150 & 46 & 5 & $1+$ \\
2 & Female & Left & 64 & 167 & 31 & 1 & 2 \\
\hline 3 & Male & Left & 74 & 176 & 65 & 4 & 2 \\
\hline 4 & Female & Right & 61 & 164 & 42 & 2 & $1+$ \\
\hline 5 & Female & Left & 63 & 159 & 47 & 3 & 2 \\
\hline 6 & Female & Left & 60 & 165 & 45 & $1 / 5$ & 2 \\
\hline Mean and SD & - & - & $62 / 8 \pm 6 / 3$ & $1 / 60 \pm 0 / 08$ & $62 / 8 \pm 6 / 3$ & $2 / 75 \pm 1 / 54$ & - \\
\hline
\end{tabular}

Table 2. Mean and SD of walking parameters with three different types of AFO

\begin{tabular}{ccccc} 
& \multicolumn{2}{c}{ Walking condition } & & \\
& Ankle neutral full & Ankle neutral 3/4 & Ankle (5 D.F)full & P-value \\
& foot plate & foot plate & foot plate \\
\hline Walking velocity & $0.53 \pm 0.10$ & $0.61 \pm 0.15$ & $0.59 \pm 0.11$ & $0.83 \pm 0.07$ \\
Stride length & $0.76 \pm 0.12$ & $0.82 \pm 0.08$ & $0 / 164$ \\
Cadence & $44 \pm 11.09$ & $45 \pm 11.07$ & $44 \pm 8.31$ & $1 / 000$ \\
\hline $\begin{array}{c}\text { Maximum knee } \\
\text { extension }\end{array}$ & $-6.42 \pm 8.04$ & $-6.12 \pm 10.09$ & $-3.9 \pm 9.94$ & $0 / 342$
\end{tabular}

\section{Discussion}

The stroke affects many aspects of the person's life, but the main problems of these individuals are gait pattern disorders. Insufficient ankle dorsiflexion in the swing phase, medio-lateral ankle instability, and inefficient foot-off during the terminal stance phase causes asymmetric gait pattern and reduced walking speed in these patients and an AFO is often prescribed to improve gait (Ohsawa, et al., 1992). A forefoot strike at the early stance phase, knee genurecurvatum and abnormal knee hyperextension (greater than 5 degrees) are features those could transferring the ground reaction force vector to the anterior knee axis from the mid to late stance phase and can be observed in such patients, which also followed by uncoordinated gait pattern and reduced walking speed. The AFO is able to control hyperextension by alteration the place of the ground reaction force vector and correct inadequate dorsiflexion. Various studies have showed the positive effects of AFO on the gait parameters in the hemiplegic individuals. Depending on the structure of AFO, the plantar flexor muscle activity and knee stability can be improved by using this orthosis. The current study examined the effect of ankle alignment and footplate length of rigid AFO on the spatiotemporal parameters and maximum knee extension in the stance phase during single limb support in people with post stroke hemiplegia.

According to findings from this study, the walking speed of patients with $5^{\circ} \mathrm{DF}$ AFO was increased to $11.56 \%$, which is statistically significant compared to CAFO. This increase is probably due to the ankle dorsiflexion angle, which may help to recreate real heel strike, control the progression of the tibia from the mid to late stance phase, forward anterior thigh movement, positioning the limb in a better alignment for weigh distribution to the opposite limb and start the better swing phase in the same direction (Lehmann, et al., 1987). Improvement in the walking speed with AFO is also associated with the tibia inclination 
(leg angle relative to the vertical angle) (Fatone, et al., 2009). The result of this section of our study was in line with the study of Lehman et al, but inconsistent with Barbara Silver et al. (2011). They stated that the walking speed in dynamic AFO in two different states of neutral AFO and $5^{\circ} \mathrm{DF}$ AFO did not differ statistically. In addition, it is possible to make meaningful changes by increasing the sample size and increasing the dorsiflexion angle up to 10 degrees (SilverThorn, Herrmann, Current, \& McGuire, 2011). In the $3 / 4 \mathrm{AFO}$, almost all patients showed an increase in the walking speed, although they were not statistically significant. Meanwhile, the ability of body to move on weight bearing limb and fast weigh distribution is of the important factor affecting the walking speed. It seems that the $3 / 4 \mathrm{AFO}$ allowed more dorsiflexion in mid foot; and the third rocker was fulfilled easier during the terminal stance phase (Goldie, Matyas, \& Evans, 1996).

In this study, there was no statistically significant difference for the stride length and the cadence between the three different conditions. Concerning the stride length, little differences were observed in both $5^{\circ} \mathrm{DF} \mathrm{AFO}$ and $3 / 4 \mathrm{AFO}$ compared to CAFO. Since increased speed is directly related to the increase in stride length (Leung \& Moseley, 2003), thus it might be considered that increased speed in 5 degree DF AFO probably was due to increased stride length.

In the $3 / 4 \mathrm{AFO}$, the barrier for push off in the terminal stance phase has been probably reduced and the movement has been made smoother, but the movement was slower in the full-length AFO due to the rigidity of the orthosis in forefoot part. There was no significant change in stride length in comparison to the $5^{\circ} \mathrm{DF} A F O$ and the CAFO in our study, which is in the line with Barbara Silver et al. study (Silver-Thorn, et al., 2011). There were a few similar studies in this field and little research has been done. Concerning the cadence in relation of CAFO and the $5^{\circ} \mathrm{DF} \mathrm{AFO}$, results showed that it was consistent with the study of Barbara Silver et al.(Silver-Thorn, et al., 2011). In a review study, Leung et al. also observed no significant changes in the cadence variable through various
AFO studies (Leung \& Moseley, 2003). Luiz et al. compared the effect of different types of AFO on the cadence of stroke individuals in a review study. The hemiplegic patients commonly showed a significant improvement in walking speed without changes in cadence with the orthoses (Ferreira et al., 2013). Although, most of these studies measured short-term effects and probably different results may be obtained by examining long-term effects.

Based on the results in this study, the mean maximum knee extension angles in the stance phase during single limb support showed a decrease of $38.47 \%$ with $5^{\circ} \mathrm{DF} \mathrm{AFO}$ in comparison with $\mathrm{CAFO}$, which was not statistically significant. This reduction in the mean maximum knee extension angles in the stance phase was seen in all subjects with $5^{\circ} \mathrm{DF}$ AFO. According to the findings, it was possible that the knee was indirectly affected by the change in the ankle angle in the rigid AFO, so that the creation of the dorsiflexion and consequently the alteration of the ground reaction force vector toward the posterior knee caused a decrease in the maximum knee extension angle. Lee et al. stated that the dorsiflexion in AFO causes an increase in knee flexion in the mid stance phase, but the changes were significant when applying angles greater than 10 degrees (Lee, Lee, \& Kim, 2015). In the study of Silver-Thorn et al., the alignment of 5 degrees of dorsiflexion in the ankle caused an increase in maximum knee flexion in the loading response phase, but not statistically significant (Silver-Thorn, et al., 2011). In another study, adding below-heel wedge had better results for controlling the hyperextension with rigid AFO. In this way, it is possible that the below-heel wedge changes the angle of tibia (creating dorsiflexion), thereby causing the alteration of the ground reaction force vector and controlling the hyperextension.

It was hypothesized that $3 / 4 \mathrm{AFO}$, through the creation of dorsiflexion in the mid stance phase can cause shift of ground reaction force vector toward posterior knee and control the hyperextension. Our result of the study in relation of $3 / 4 \mathrm{AFO}$ was consistent with the study by Fatone et al., because the rigid AFO 
footplate length $(3 / 4 \mathrm{AFO})$ had no significant effect the stance knee angle (Fatone, et al., 2009). Nevertheless, additional studies with a larger sample are required to examine the influence of the AFO footplate length on the gait variables.

The short time investigation for evaluating the impact of these AFO due to the time limit for project implementation and very limited studies in relation to the AFO footplate length were our research limitations. It is suggested that the kinetics parameters (such as knee joint moment and power) and muscle activity be studied in a similar study with a larger sample, investigating the long-term effects of orthoses on gait parameters and comparing the effects of different types of articulated and non-articulated AFO in future studies.

\section{Conclusion}

According to the results of this study, it was observed that the change in the ankle angle in rigid AFO in the sagittal plane had a greater effect on the study parameters compared to the footplate length changes. Increasing the walking speed was related to altering the ankle angle, indicating the importance of the dor-

\section{References}

Bovonsunthonchai, S., Hiengkaew, V., Vachalathiti, R., \& Vongsirinavarat, M. (2011). Gait symmetrical indexes and their relationships to muscle tone, lower extremity function, and postural balance in mild to moderate stroke. Journal of the Medical Association of Thailand, 94(4), 476.

Chen, C., Yeung, K., Wu, S., \& Chiang, P. (1998). Effects of an anterior ankle-foot orthosis on hemiplegic gait. $\mathrm{J}$ Rehabil Med Assoc ROC, 26, 119-127.

da Cunha Jr, I. T., Lim, P. A., Qureshy, H., Henson, H., Monga, T., \& Protas, E. J. (2002). Gait outcomes after acute stroke rehabilitation with supported treadmill ambulation training: a randomized controlled pilot study. Archives of physical medicine and rehabilitation, 83(9), 1258-1265.

Danielsson, A., \& Sunnerhagen, K. S. (2004). Energy expenditure in stroke subjects walking with a carbon com- siflexion angle in the rigid AFO and its effect on controlling the progression of tibia and positioning the limb in more appropriate alignment. However, these changes had no significant effects on the stride length and cadence of the patients. Concerning the maximum knee extension angle in the stance phase, this change at AFO can also indirectly affect the knee and help control the hyperextension by alteration of the ground reaction force vector. Regarding the footplate length, the parameters of stride length, walking speed, cadence and maximum knee extension angle in the stance phase showed no significant changes.

\section{Acknowledgement}

The authors would like to thank all the participants in the present study. We are grateful to the University of Social Welfare and Rehabilitation Sciences for providing the equipment.

\section{Conflict of Interest Statement}

All the authors declare that they have no conflict of interest.

posite ankle foot orthosis. Journal of rehabilitation medicine, 36(4), 165-168.

Easton, J. D., Saver, J. L., Albers, G. W., Alberts, M. J., Chaturvedi, S., Feldmann, E., Kidwell, C. S. (2009). Definition and evaluation of transient ischemic attack: a scientific statement for healthcare professionals from the American Heart Association/American Stroke Association Stroke Council; Council on Cardiovascular Surgery and Anesthesia; Council on Cardiovascular Radiology and Intervention; Council on Cardiovascular Nursing; and the Interdisciplinary Council on Peripheral Vascular Disease: the American Academy of Neurology affirms the value of this statement as an educational tool for neurologists. Stroke, 40(6), 2276-2293.

Eddison, N., Chockalingam, N., \& Osborne, S. (2015). Ankle foot orthosis-footwear combination tuning: An investigation into common clinical practice in the United Kingdom. Prosthetics and orthotics international, 39(2), 
126-133.

Fatone, S., Gard, S. A., \& Malas, B. S. (2009). Effect of ankle-foot orthosis alignment and foot-plate length on the gait of adults with poststroke hemiplegia. Archives of physical medicine and rehabilitation, 90(5), 810-818.

Ferreira, L. A. B., Neto, H. P., Christovão, T. C. L., Duarte, N. A., Lazzari, R. D., Galli, M., \& Oliveira, C. S. (2013). Effect of ankle-foot orthosis on gait velocity and cadence of stroke patients: a systematic review. Journal of physical therapy science, 25(11), 1503-1508.

Goldie, P. A., Matyas, T. A., \& Evans, O. M. (1996). Deficit and change in gait velocity during rehabilitation after stroke. Archives of physical medicine and rehabilitation, 77(10), 1074-1082.

Hsu, J. D., Michael, J., \& Fisk, J. (2008). AAOS Atlas of Orthoses and Assistive Devices E-Book: Elsevier Health Sciences.

Hung, J.-W., Chen, P.-C., Yu, M.-Y., \& Hsieh, Y.-W. (2011). Long-term effect of an anterior ankle-foot orthosis on functional walking ability of chronic stroke patients. American journal of physical medicine \& rehabilitation, 90(1), 8-16.

Lamontagne, A., Malouin, F., \& Richards, C. L. (2001). Locomotor-specific measure of spasticity of plantarflexor muscles after stroke. Archives of physical medicine and rehabilitation, 82(12), 1696-1704.

Lee, H. Y., Lee, J. H., \& Kim, K. (2015). Changes in angular kinematics of the paretic lower limb at different orthotic angles of plantar flexion limitation of an anklefoot-orthosis for stroke patients. Journal of physical therapy science, $27(3), 825-828$.

Lehmann, J. F., Condon, S. M., \& Price, R. (1987). Gait abnormalities in hemiplegia: their correction by anklefoot orthoses. Archives of Physical Medicine and Rehabilitation, 68(11), 763-771.

Leung, J., \& Moseley, A. (2003). Impact of ankle-foot orthoses on gait and leg muscle activity in adults with hemiplegia: systematic literature review. Physiotherapy, 89(1), 39-55.

Miyazaki, S., Yamamoto, S., \& Kubota, T. (1997). Effect of ankle-foot orthosis on active ankle moment in patients with hemiparesis. Medical and Biological Engineering and Computing, 35(4), 381-385.

Ohsawa, S., Ikeda, S., Tanaka, S., Takahashi, T., Takeuchi, T., Utsunomiya, M., . . Katagi, Y. (1992). A new model of plastic ankle foot orthosis (FAFO (II)) against spastic foot and genu recurvatum. Prosthetics and orthotics international, 16(2), 104-108.

Silver-Thorn, B., Herrmann, A., Current, T., \& McGuire, J. (2011). Effect of ankle orientation on heel loading and knee stability for post-stroke individuals wearing anklefoot orthoses. Prosthetics and orthotics international, $35(2), 150-162$. 


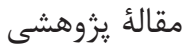

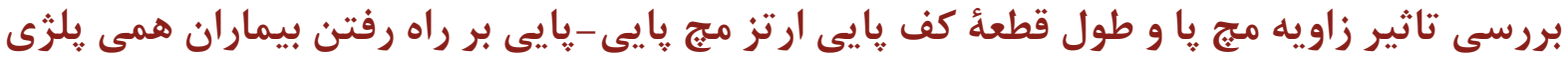 دجار سكته مغزى
}

\author{
راضيه ديده ورا'، غلامرضا امينيان"'، عاليه دريابر \\ ا. دانشجو كارشناسى ارشد، كروه آموزشى ارتز و يروتز، دانشكاه علوم بهزيستى و توانبخشى، تهران، ايران

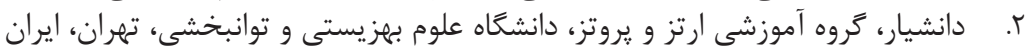

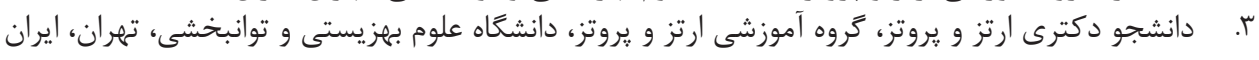

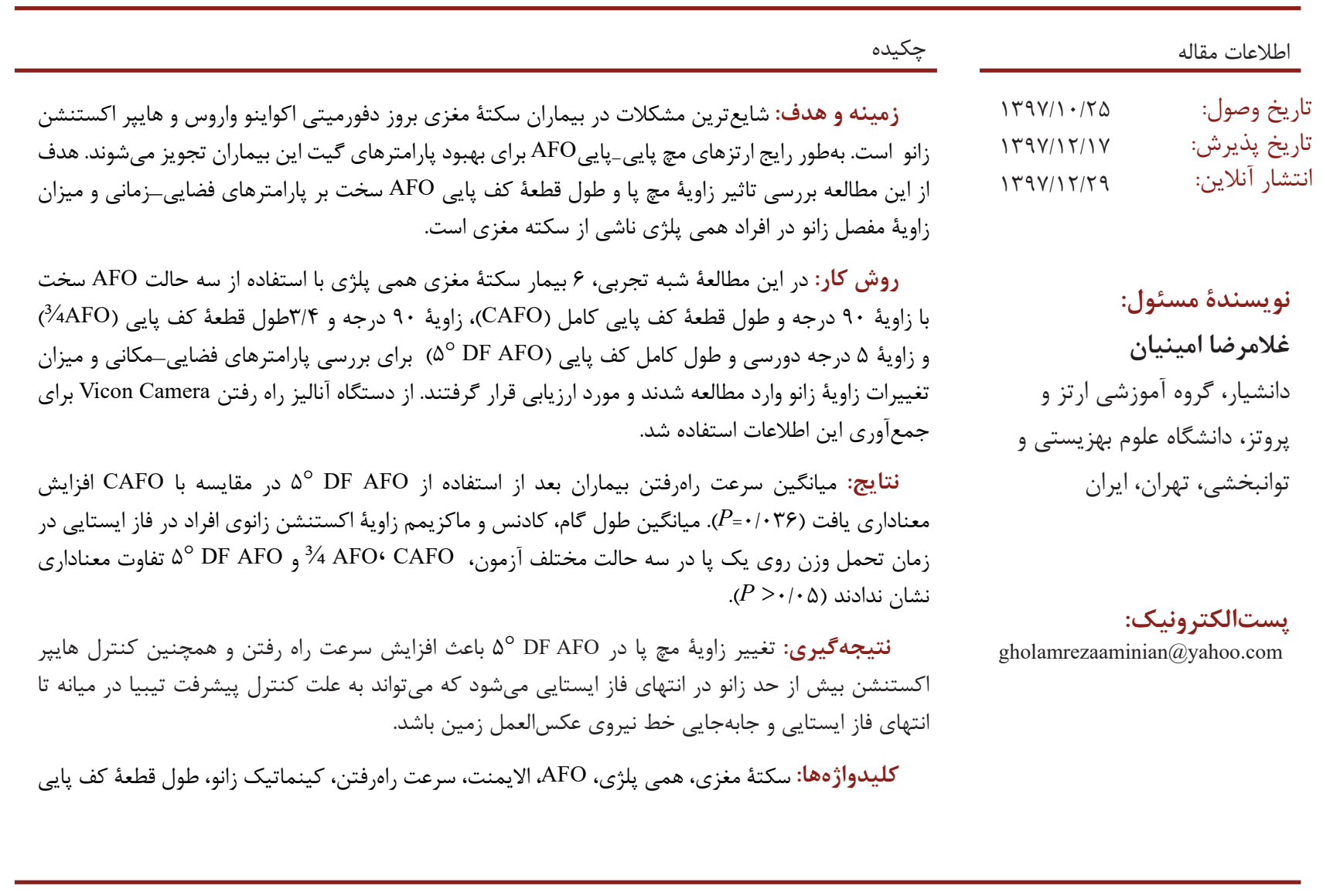

